\title{
Site-specific integration of bacteriophage VWB genome into Streptomyces venezuelae and construction of a VWB-based integrative vector
}

\author{
Lieve Van Mellaert, Lijuan Mei, Elke Lammertyn, Sabine Schacht \\ and Jozef Anné
}

Author for correspondence: Jozef Anné. Tel: +32 163373 75. Fax: +32 16337340 . e-mail: Jozef.Anne@rega.kuleuven.ac.be

Laboratory of Bacteriology, Rega Instituut, KU Leuven, Minderbroedersstraat 10 B-3000 Leuven, Belgium
The temperate bacteriophage VWB integrates into the chromosome of Streptomyces venezuelae ETH14630 via site-specific integration. Following recombination of the VWB attP region with the chromosomal attB sequence, the host-phage junctions att $L$ and att $R$ are formed. Nucleotide sequence analysis of attP, attB, attL and attR revealed a 45 bp common core sequence. In attB this 45 bp sequence consists of the $3^{\prime}$ end of a putative tRNA ${ }^{\text {Arg }}$ (AGG) gene with a 3'-terminal CCA sequence which is typical for prokaryotic tRNAs. Phage DNA integration restores the putative tRNA Arg(AGG) gene in attL. However, following recombination the CCA sequence is missing as is the case for most Streptomyces tRNA genes described so far. Adjacent to VWB attP, an ORF encoding a 427 aa protein was detected. The C-terminal region of this protein shows high similarity to the conserved C-terminal domain of site-specific recombinases belonging to the integrase family. To prove the functionality of this putative integrase gene (int), an integrative vector pKT02 was constructed. This vector consists of a $2.3 \mathrm{~kb}$ HindIII-Sphl restriction fragment of VWB DNA containing attP and int cloned in a non-replicative Escherichia coli vector carrying a thiostrepton-resistance (tsr) gene. Integration of pKT02 was obtained after transformation of Streptomyces venezuelae ETH14630 and Streptomyces lividans TK24 protoplasts. This vector will thus be useful for a number of additional Streptomyces species in which a suitable tRNA gene can be functional as integration site.

Keywords: Streptomyces, bacteriophage VWB, integration, tRNA, integrative vector

\section{INTRODUCTION}

The temperate phage VWB was originally isolated from soil using Streptomyces venezuelae ETH14630 as indicator strain (Anné et al., 1984). Its dsDNA genome is $47 \cdot 3 \mathrm{~kb}$ in size and has a GC content of $69 \cdot 3 \mathrm{~mol} \%$. VWB DNA has cohesive ends, implying a site-specific staggered cutting of concatemeric DNA during DNA packaging. Phages with such a non-headful packaging system are suitable as cloning vectors as reported, e.g. $\phi$ C31 (Hopwood et al., 1987) and phage VWB. The latter phage can package at least $3.5-4 \mathrm{~kb}$ foreign DNA in addition to its own genome (Anné et al., 1990a).

Streptomyces integrative plasmids and temperate phages

The EMBL accession numbers for the sequences reported in this paper are AJ000047-AJ000050. (including two plasmids of Saccharopolyspora erythraea, previously classified as Streptomyces erythraeus) most often integrate site-specifically into the host chromosome (Bar-Nir et al., 1992; Boccard et al., 1988; Brasch et al., 1993; Brown et al., 1990, 1994; Gabriel et al., 1995; Kendall \& Cullum, 1986; Kuhstoss \& Rao, 1991; Shirai et al., 1991; Sosio et al., 1989). In general, such site-specific integration is catalysed by a sitespecific recombinase. Streptomyces integrative plasmids encode a protein belonging to the integrase family showing similarities with the phage $\lambda$ integrase. Streptomyces phages have different types of proteins that cause site-specific integration. For example, Streptomyces phage $\phi \mathrm{C} 31$ encodes p68 (Kuhstoss \& Rao, 1991), which could be aligned to the resolvase/DNA invertase family of site-specific recombinases (Leschziner et al., 1995). In phage R4, sre encodes a protein similar to the resolvase/ invertase family (Matsuura et al., 1996). The lysogenic 
Streptomyces rimosus RP3 phage requires a phageencoded protein belonging to the integrase family (Gabriel et al., 1995).

Integration of phages and plasmids of Streptomyces which utilize an Int family recombinase occurs into a tRNA gene (Bar-Nir et al., 1992; Brown et al., 1990, 1994; Mazodier et al., 1990; Reiter et al., 1989; Vögtli \& Cohen, 1992). attP contains the $3^{\prime}$ end of the tRNA gene so that after the recombination event the chromosomal tRNA is restored in attL.

Phage VWB has a narrow host range, i.e. among six $S$. venezuelae strains, only the ETH14630 strain in addition to Streptomyces exfoliatus ATCC 12672 could be infected. Nevertheless, VWB could be introduced by transfection into several non-infectible Streptomyces strains, including Streptomyces lividans, with the formation of lysogens (Anné et al., 1990b). Bacteriophage VWB integrates site specifically into the chromosome of its host S. venezuelae ETH14630 at the attachment site attB (Anné et al., 1990b).

In this study, the att sites involved in the integration of VWB into the $S$. venezuelae ETH14630 chromosome were characterized and an ORF (int) upstream of attP encoding a 427 aa protein resembling the recombinases of the integrase family (Argos et al., 1986; Abremski \& Hoess, 1992; Esposito \& Scocca, 1997; Nunes-Düby et al., 1998) was identified. By introducing deletions in the int gene, we proved the requirement of the gene for sitespecific recombination of the attP with the attB site. Furthermore, a functional integrative vector was constructed using an Escherichia coli replicon containing the VWB attP site, the int gene and a thiostreptonresistance $(t s r)$ marker.

\section{METHODS}

Bacterial strains, plasmids, growth conditions and transformation experiments. S. venezuelae ETH14630 was used as host strain for phage VWB propagation (Anné et al., 1984). Integration of VWB DNA or VWB-based vectors was tested both in S. venezuelae ETH14630 and in S. lividans TK24 (Hopwood et al., 1985), grown in phage medium (Korn et al., 1978) supplemented, when necessary, with $10 \mu \mathrm{g}$ thiostrepton $\mathrm{ml}^{-1}$. Protoplast transformation was carried out as previously described (Anné et al., 1990c; Hopwood et al., 1985). Regeneration of protoplasts and growth occurred on MRYE medium (Anné et al., 1990c) and transformants were selected by means of an overlay with $2.5 \mathrm{ml}$ soft MRYE medium containing 75,150 or $300 \mu \mathrm{g}$ thiostrepton $\mathrm{ml}^{-1} 20-24 \mathrm{~h}$ after transformation.

A genomic bank of S. venezuelae or its VWB lysogen was made in E. coli MC1061 (Wertman et al., 1986) using pACYC184 (Chang \& Cohen, 1978) as cloning vector. E. coli TG1 (Sambrook et al., 1989) was used for subcloning of DNA fragments into pUC18/19 (Yanisch-Perron et al., 1985) or pBluescriptIIKS $(+)$ (Stratagene). E. coli strains were grown in LB medium, and when applicable, ampicillin $\left(50 \mu \mathrm{g} \mathrm{ml}^{-1}\right)$ or chloramphenicol $\left(12.5 \mu \mathrm{g} \mathrm{ml}^{-1}\right)$ was added.

DNA manipulations. Total DNA of Streptomyces cells was isolated as described by Hopwood et al. (1985). Plasmid DNA isolation from $E$. coli cells, restriction enzyme digestions and other DNA manipulations were carried out by standard protocols (Sambrook et al., 1989). Restriction DNA fragments were eluted from agarose gels using the QIAquick Gel Extraction Kit (QIAGEN).

Hybridization experiments. To identify the attP region, $2 \mu \mathrm{g} S$. venezuelae DNA, $2 \mu \mathrm{g}$ lysogenic DNA and $0.5 \mu \mathrm{g}$ VWB DNA were digested with different restriction enzymes. The DNA fragments were subsequently separated by agarose gel electrophoresis and blotted on Hybond-N membrane (Amersham) using a VacuGene system (Pharmacia). The UV cross-linked DNA fragments were hybridized with an appropriate digoxigenin-labelled probe as described by Engler-Blum et al. (1993). Hybridization signals were detected with $0.25 \mathrm{mM}$ CDP-Star (Tropix) according to the method of Hoeltke et al. (1995).

To isolate $a t t B$, att $L$ and $a t t R$, a genomic bank of $S$. venezuelae and of its VWB lysogen was made following digestion of the chromosomal DNA with $S p h I$ and $B c l$, respectively. The digested DNA was subsequently ligated to $S p h \mathrm{I}$ - or BamHIdigested pACYC184. After transformation of E.coli MC1061, colonies were lifted on Hybond $-\mathrm{N}^{+}$membranes (Amersham) following the manufacturer's instructions. Next, a digoxigenin-labelled attP-specific probe was used for selection of the desired subclones.

To demonstrate the integration of the VWB-based integration vector into the $S$, venezuelae or $S$. lividans chromosome, $2 \mu \mathrm{g}$ chromosomal DNA isolated from strains with or without the integrated vector, and $1 \mathrm{ng}$ vector were digested and blotted on Hybond- $\mathrm{N}$ membrane as described above. The digoxigenin-labelled $0.5 \mathrm{~kb}$ Bam HI-HindII fragment of VWB containing the attP locus was used as probe.

Sequencing and computer-assisted analyses. Nucleotide sequence analysis was performed by manual sequencing using $\left[{ }^{35}\right.$ S]dATP $\alpha$ S (NEN Life Science Products) or by automated sequencing on ALFexpress (Pharmacia) using CY5-labelled oligonucleotides. Sequencing was repeatedly carried out on both strands. Computer-assisted analyses of sequences occurred with programs of PC/GENE (Genofit, IntelliGenetics)

Construction of the integrative vector. For the construction of the integrative vector pKT02 (see Fig. 6), E. coli vector pIC20R (Marsh et al., 1984) was used, in which the $t s r$ gene was cloned by inserting the $1.1 \mathrm{~kb} \mathrm{Bcll}$ fragment of pIJ702 (Katz et al., 1983) into the BamHI site. The resulting pKTO1 vector was linearized with PstI, blunted in a T4 DNA polymerase reaction, next digested with HindIII and finally ligated to the $2.3 \mathrm{~kb} S p h \mathrm{I}$ (sticky ends removed by T4 DNA polymerase)-HindIII VWB fragment. pKT02 thus contained the $t s r$ gene, the attP locus and the int gene of VWB.

In addition, two derivatives of pKT02 were made. In pKT03, the $1.6 \mathrm{~kb}$ HindIII-BamHI fragment (Fig. 1) containing the $3^{\prime}$ terminal part of the int gene was removed. In pKT04, a $0.6 \mathrm{~kb}$ internal PstI fragment of the int gene (Fig. 1) was deleted so that only the first $265 \mathrm{~N}$-terminal aa of the integrase protein remained.

\section{RESULTS AND DISCUSSION}

\section{Characterization of attP and int gene of VWB}

In a previous report, attP of phage VWB was localized on the left side of the $9.6 \mathrm{~kb} S p h \mathrm{I}-\mathrm{B}$ fragment (Anné et al., 1990b). Further comparisons between hybridization patterns of phage VWB and its prophage, using more sensitive methods for the detection of hybridizing bands, revealed that attP is located within the $1.9 \mathrm{~kb}$ VWB 
(a)

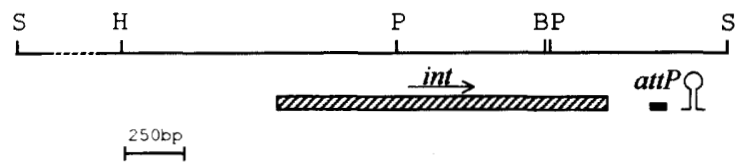

(b)

pKT02

pKT03

рKT04 integration

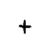

$-$
Fig. 1. (a) Schematic representation of the Sphl-B fragment of the WWB genome which contains the int gene (खmos) and attP locus (a), followed by a very stable stemloop structure ( () . The relevant restriction sites are indicated $(B, B a m H I ; H$, Hindill; $P$, Pstl; S, Sphi). (b) Fragments cloned in pKT01 to investigate the functionality of the int gene and resulting in integration of the integrative vector $(+)$ or $\operatorname{not}(-)$.

(a)

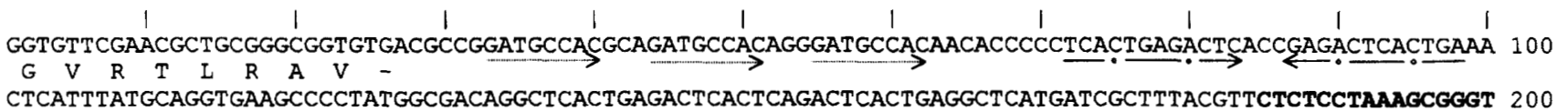

CTCATTTATGCAGGTGAAGCCCCTATGGCGACAGGCTCACTGAGACTCACTCAGACTCACTGAGGCTCATGATCGCTTTACGTTCTCTCCTAAAGCGGGT 200

GTCGCAGGTTCGAATCCTGCCGGGGGACAACCTGCATCGCAGGTCAGGGAGTCAACGGCCCCCCCGTTCCATTCGAACGGGGGGGCCGTTGTCGTACCC 300

GGATGCCACATAGATGCCACATCCCCACGGAATCCTGCGGATCACGTCGCTCGAAAGAGTGATGTGCACAGCAGCCACAATGCGTAGAGTGCTGTCTACC 400

(b)

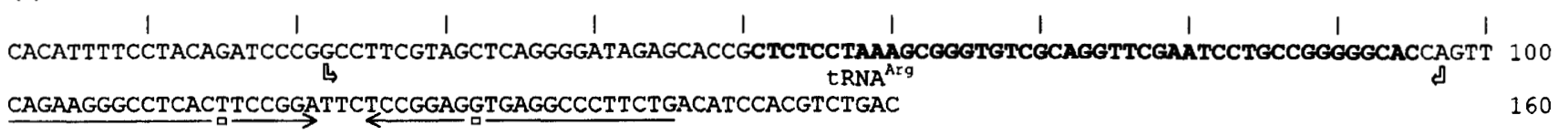

Fig. 2. (a) Nucleotide sequence downstream of the int gene containing the attP sequence. The identical sequence is given in bold characters. The C-terminal amino acid sequence of Int is shown below the int sequence. Arrows under the sequence show direct and indirect repeats and mismatches within the repeats are indicated by small circles. (b) Nucleotide sequence of the $S$. venezuelae att $B$ region with the common sequence indicated by bold characters. The tRNA Arg gene is indicated by open arrows. Arrows under the sequence show an inverted repeat with one allowed G-U bp (־).

BamHI-H1 restriction fragment. Since both the SphI-B and the BamHI-H1 VWB restriction fragments contained the attP locus, it could be localized on the common part of these fragments, i.e. a $0.7 \mathrm{~kb} \mathrm{BamHI-}$ $S p h I$ fragment that is on the right side of the SphI-B fragment (Fig. 1). Sequencing this $0.7 \mathrm{~kb}$ fragment revealed an incomplete ORF encoding a polypeptide sequence that could be aligned to the conserved Cterminal domain of the recombinases of the integrase family (Argos et al., 1986; Esposito \& Scocca, 1997; Nunes-Düby et al., 1998). The ORF is followed by three direct repeats of $8 \mathrm{bp}$ of which the function is unknown and one inverted repeat of $12 \mathrm{bp}$ with two mismatches (Fig. 2a). This inverted repeat may serve as a transcriptional terminator of the ORF. Approximately $170 \mathrm{bp}$ downstream of the TGA stop codon, a sequence with similarities to the 3' end of known tRNA genes (Sprinzl et al., 1991) was found. tRNA genes are frequently utilized as attachment sites for integrative elements (Reiter et al., 1989) and this sequence was therefore presumed to be part of the VWB attP locus. A perfect inverted repeat composed of $17 \mathrm{bp}$ arms interrupted by five unpaired bases followed this putative attP sequence
(Fig. 2). The free energy $\Delta G\left(25^{\circ} \mathrm{C}\right)$ of the stem-loop for the RNA molecule is calculated to be $-50.8 \mathrm{kcal} \mathrm{mol}^{-1}$ $\left(-212.5 \mathrm{~kJ} \mathrm{~mol}^{-1}\right)$.

Further sequencing of the ORF (located on the upstream $1.6 \mathrm{~kb}$ HindIII-BamHI fragment, Fig. 1) revealed a complete ORF of $1284 \mathrm{nt}$ encoding a putative integrase (Int). The int start codon is an ATG, which is preceded by a Shine-Dalgarno sequence (AGGTGA). The int gene can encode a protein of 427 aa with a deduced molecular mass of $49.1 \mathrm{kDa}$. This protein displays the features of an integrase as concluded from the homology of its C-terminal region to recombinases of the integrase family (Argos et al., 1986; Esposito \& Scocca, 1997; Nunes-Düby et al., 1998).

Although this family exhibits a large diversity of sequences, within the C-terminal catalytic domain two Arg residues and a Tyr residue are absolutely conserved whilst a His residue is highly conserved. These four conserved residues form the active site and can be placed in three boxes ( $A, B$ and $C$ ) showing high similarity among nearly all proteins of the integrase family (Esposito \& Scocca, 1997). Recently, Nunes-Düby et al. 


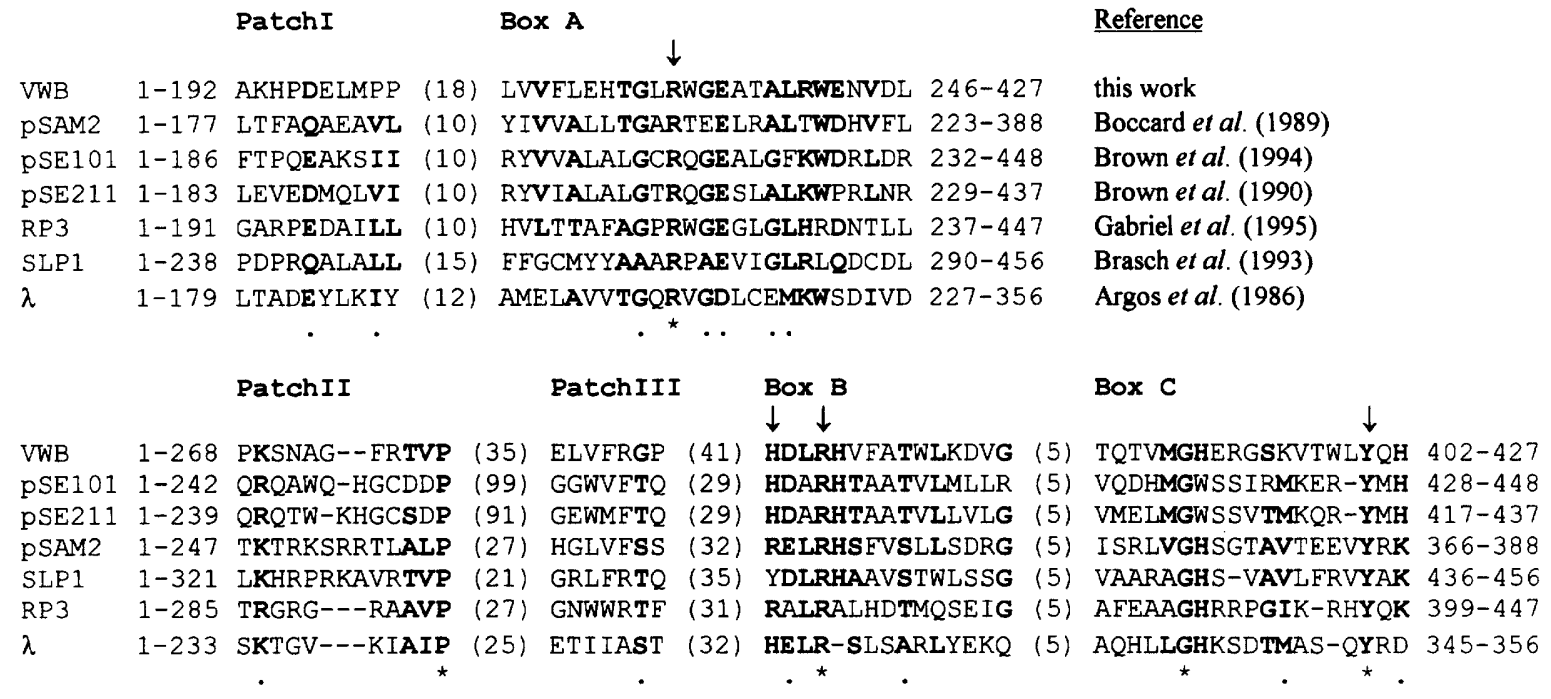

Fig. 3. Alignment of amino acid sequences of six domains of the WWB integrase protein with amino acids in the same domains of the $\lambda$ Int, the prototypical member of the integrase family, and other Streptomyces integrases. Boxes A, B and $C$ correspond to the three major clusters with global similarities among the proteins of the integrase family described by Esposito \& Scocca (1997). Patches I, II and III refer to the three additional patches of conserved sequence among the integrase family proteins found by Nunes-Düby et al. (1998). The numbers of amino acids spanning the different domains are indicated by the numbers in parentheses. Bold characters indicate similar or identical residues at a particular position in five of the seven aligned integrase proteins; asterisks indicate perfectly conserved residues and dots indicate wellconserved residues among the aligned proteins. The arrows indicate the four active-site residues of the integrases.

(1998) identified three additional sequence patches (I, II and III). The Tyr is involved in the formation of a phosphotyrosyl bond to the cleaved $3^{\prime}$ end of the DNA. This covalent protein-DNA linkage conserves the bond energy, which is later transferred to the DNA during ligation. The three other active-site residues Arg, His and Arg form a basic triad and are structurally clustered in the active-site cleft as shown by crystal structure analysis of four integrase proteins (Yang \& Mizuuchi, 1997). In the VWB Int protein, the four conserved residues all are present and its $\mathrm{C}$-terminal sequence can be aligned (Fig. 3) to the mentioned boxes and patches found in the catalytic domain of the proteins of the Int family. In patch I a group of acidic amino acids and precisely spaced hydrophobic residues, LT-EEV--LL, are conserved. The VWB Int sequence, however, shows in this domain rather poor similarity with the described consensus sequence. In box A, the first conserved Arg residue is found together with the highly conserved Gly and Glu residues, at positions 231, 229 and 234, respectively. In patch II, a conserved Lys residue is involved which is flanked by Ser or Thr in one subgroup of Int family proteins and by Gly or Met in the other subgroup. In VWB Int the corresponding $\mathrm{Lys}_{270}$ is indeed followed by a Ser, but preceded by a Pro. The hydrophobic Phe-rich cluster found in patch III is represented by $\mathrm{Leu}_{316}-\mathrm{Val}_{317}-\mathrm{Phe}_{318}$. The acidic amino acids mostly preceding this cluster are $\mathrm{Asp}_{314}$ and $\mathrm{Glu}_{315}$. The polar residues following the hydrophobic stretch in the majority of Int family proteins are not present in VWB Int. Box B includes the His-X-Leu-Arg-His motif (residues 363-367) of which the first His and the Arg are active-site residues. Box $\mathrm{C}$ contains, besides the active- site Tyr (position 400), two highly conserved aa, namely $\mathrm{Gly}_{389}$ and $\mathrm{His}_{390}$. They belong to the Leu-Leu-Gly-His consensus sequence found in this domain. The spacing between the two conserved Arg residues in VWB Int consists of 136 aa, which is rather long in comparison with other members of the integrase family. The distance between the second conserved Arg and the active-site Tyr is 32 aa as in the majority of the proteins analysed by Esposito \& Scocca (1997).

Furthermore, Int has a calculated pI value of $10 \cdot 2$. The basic protein contains $20.3 \%$ basic amino acids and only $12 \cdot 6 \%$ acidic residues, which is typical for DNA-binding proteins. In addition, int is located close to the attP locus as found for most other integrases.

For many int genes described, the ORF is immediately preceded by or overlaps an ORF which is defined in most instances as, or speculated to be, the excisionase (xis) gene. Up to now, no ORF sharing similarity with the described Streptomyces excisionase proteins (Boccard et al., 1989; Brasch et al., 1993; Brown et al., 1990, 1994; Gabriel et al., 1995) could be detected in the sequence surrounding VWB int.

It is remarkable that all Streptomyces plasmids that can integrate into the host chromosome code for an integrase belonging to the Int class of site-specific recombinases. The use of a site-specific recombinase of the integrase family is, however, not common for Streptomyces phages. Like VWB, RP3 encodes an integrase (Gabriel et al., 1995), but $\phi \mathrm{C} 31$ and $\mathrm{R} 4$ use a recombinase with a resemblance to the proteins of the resolvase/invertase family (Leschziner et al., 1995; Matsuura et al., 1996). 
att CGGCCTTCGTAGCTCÀGGGGATAGAGCACCGCTCTCCTAAAGCGGGTGTCGCAGGTTCGAATCCTGCCGGGGGCACCAGTTCAG att $L$ CGGCCTTCGTAGCTCA'GGGGATAGAGCACCGCTCTCCTAAAGCGGGTGTCGCAGGTTCGAATCCTGCCGGGGGCACAACCTGCA at $R$ GACTCACTGAGGCTCATGATCGCTTTACGTTCTCTCCTAAAGCGGGTGTCGCAGGTTCGAATCCTGCCGGGGGCACCAGTTCAG attP GACTCACTGAGGCTCATGATCGCTTTACGTTCTCTCCTAAAGCGGGTGTCGCAGGTTCGAATCCTGCCGGGGGCACAACCTGCA

Fig. 4. Alignment of the att loci involved in site-specific recombination of the VWB genome into the $S$. venezuelae chromosome. The 45 bp identical sequence is presented in bold characters and the tRNA ${ }^{\text {Arg }}$ genes found both in attB and attL are boxed. Arrows represent the two types of inverted repeats seen at the $5^{\prime}$ end of the common att region. Dotted lines mark the small 5 bp identical box.

\section{Nucleotide sequence of attB, attL and attR}

Phage VWB integrates into its host chromosome by recombination with the host chromosomal attB locus. This recombination regenerates two hybrid sites, att $L$ and attR. These three att loci were isolated from genomic libraries representing wild-type and VWBlysogenized $S$. venezuelae strains. Chromosomal DNA of $S$. venezuelae and the lysogen was digested with $S p h \mathrm{I}$ and $B c l$ I, respectively. On hybridization with the digoxigenin-labelled $0.7 \mathrm{~kb}$ Bam HI-SphI VWB fragment containing the attP site, clear hybridization signals could be detected. The $S p h \mathrm{I}$ fragment of $S$. venezuelae DNA containing the attB locus was about $4 \mathrm{~kb}$; the $B c l$ I fragments with the recombinant att loci (attL, attR) were $0.8 \mathrm{~kb}$ and more than $8 \mathrm{~kb}$. These digests were ligated to pACYC184 digested with SphI or BamHI. E. coli MC1061 transformants containing DNA fragments homologous to attP were selected by colony hybridization with the $0.7 \mathrm{~kb} B a m \mathrm{HI}-S p h \mathrm{I}$ VWB fragment as probe. Positive inserts were subcloned and subsequent hybridization experiments revealed the presence of $a t t B$ on a $0.4 \mathrm{~kb} F s p \mathrm{I}-S m a \mathrm{I}$ fragment. The recombinant att loci $(a t t L, a t t R)$ were localized on a $0.5 \mathrm{~kb} \mathrm{BclI}-S p h \mathrm{I}$ and a $0.6 \mathrm{~kb}$ Drall fragment, respectively.

DNA sequences of the four att loci were aligned (Fig. 4). This alignment revealed a $45 \mathrm{bp}$ sequence common to the four att sites without duplications of the chromosomal sequences in the integrated state.

\section{VWB DNA integrates into a putative tRNA ${ }^{\text {Arg }}$ gene}

tRNA genes are often the sites at which the recombination events with bacterial integrative elements occur (Reiter et al., 1989). A tRNA search in the analysed sequences revealed the presence of a tRNA cloverleaf secondary structure in attB and att $L$ transcripts (Figs 4 and 5). The 45 bp identical sequence comprises the $3^{\prime}$ end of a tRNA ${ }^{\mathrm{Arg}}(\mathrm{AGG})$ gene with a $5^{\prime} \mathrm{CCU}$ anticodon. The tRNA ${ }^{\mathrm{Arg}}$ genes present in $a t t B$ and att $L$ differ from each other in that the CCA terminus typical for prokaryotic tRNAs is present in $a t t B$, but is lacking in attL. This CCA sequence is present in most bacterial tRNA genes (Sprinzl et al., 1991), but seems to be absent in the majority of Streptomyces tRNA genes. In these cases, the CCA terminus can be added post-transcriptionally by a nucleotidyltransferase. Only one of the 18 characterized $S$. lividans tRNA genes, i.e. cys $T$, encodes the CCA terminus as is the case for bldA [a

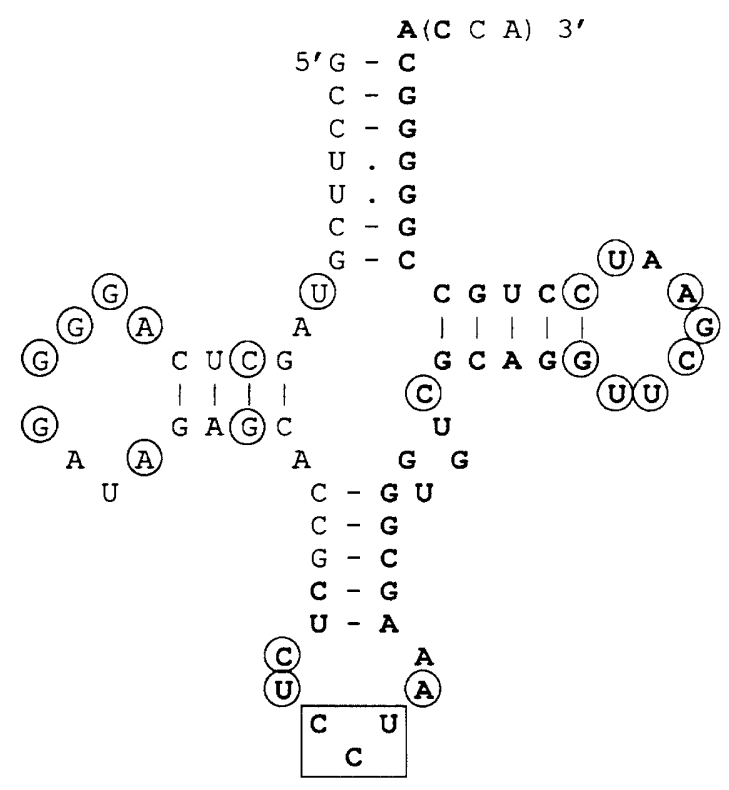

Fig. 5. Cloverleaf structure of the tRNA ${ }^{\text {Arg }}$ genes found in attB and attl. The anticodon $5^{\prime} \mathrm{CCU}$ is boxed. The nucleotides common to the attP sequence are in bold characters. Circled nucleotides correspond to conserved nucleotides in tRNAs (Sprinzl et al., 1991). The CCA sequence in parentheses is present only in the tRNA gene of attB.

tRNA ${ }^{\text {Leu }}$ (TTA) gene] from Streptomyces coelicolor and Streptomyces griseus (Sedlmeier et al., 1994).

Further experiments are required to answer the question whether the tRNA ${ }^{\text {Arg }}$ genes present in attB of $S$. venezuelae and in attL after VWB integration into the chromosome are transcribed and functional. It was shown in other reports that the integrated plasmid pSG1 is flanked at attL by a functional tRNA ${ }^{\text {Ser }}$ gene (Bar-Nir et al., 1992) and SLP1 integrates in a biologically functional tRNA ${ }^{\text {Tyr }}$ gene that is essential for host viability (Vögtli \& Cohen, 1992). The tRNA ${ }^{\text {Arg }}$ gene described in this report is followed by an energetically stable stem-loop structure in both attB and attL. The putative hairpin downstream of the tRNA ${ }^{\mathrm{Arg}}$ gene in attB has a stem size of $22 \mathrm{bp}$ with one allowed G-U base pair and a loop of 3 unpaired bases (Fig. 2b). The free energy of this structure is calculated to be $-48.8 \mathrm{kcal}$ $\mathrm{mol}^{-1}\left(-204.2 \mathrm{~kJ} \mathrm{~mol}^{-1}\right)$. The tRNA ${ }^{\mathrm{Arg} g}$ gene in att $\mathrm{k}$ is followed by the previously described hairpin that is equally present downstream of the attP locus. Both 

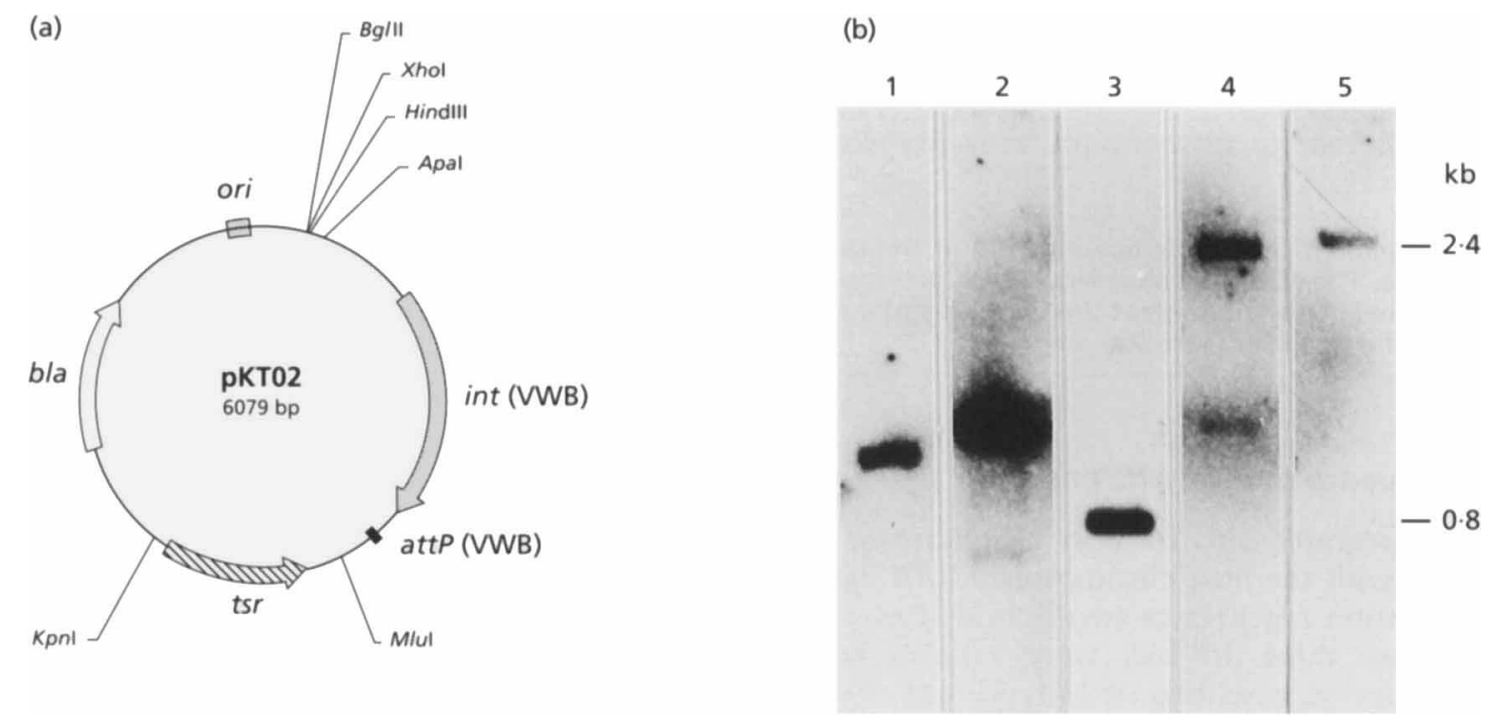

\begin{abstract}
Fig. 6. (a) A schematic map of the integrative pKTO2 vector. The ampicillin-resistance gene (b/a) is only expressed in $E$. coli. The thiostrepton-resistance gene (tsr) is used as selection marker in Streptomyces. The restriction sites indicated on the map are unique and available to clone genes of interest. (b) Site-specific integration of pKT02 in the S. venezuelae and S. lividans chromosome: $2 \mu \mathrm{g}$ Hindll-digested chromosomal DNA of S. venezuelae (lane 1), $2 \mu \mathrm{g}$ Hindll-digested chromosomal DNA of $S$. venezuelae[pKT02] (lane 2), $1 \mathrm{ng}$ Hindll-digested pKT02 (lane 3), $2 \mu \mathrm{g}$ Hindll-digested chromosomal DNA of S. lividans (lane 4) and $2 \mu \mathrm{g}$ Hindll-digested chromosomal DNA of S. lividans[pKT02] (lane 5) were blotted on membrane. The Southern blot was hybridized with the digoxigenin-labelled $0.5 \mathrm{~kb} B a m \mathrm{HI}-\mathrm{Hindll}$ fragment of VWB containing the attP locus.
\end{abstract}

putative hairpins may serve as transcription terminator indicative of functionality. After integration this could prevent transcription of phage genes which are possibly toxic for the host cell.

Besides VWB, the only Streptomyces phage known that uses an integrase to recombine its genome with the host chromosome is RP3. It also integrates in a tRNA ${ }^{\text {Arg }}$ (AGG) gene of the $S$. rimosus chromosome (Gabriel et al., 1995). Both tRNA ${ }^{\mathrm{Arg}}$ genes are identical with the exception of one nucleotide located in the more variable tRNA acceptor stem. Although the common att region of RP3 is described to be somewhat longer than that of VWB, it shares the total $45 \mathrm{bp}$ VWB attP core sequence. There are, however, no similarities in the DNA regions flanking the two attP sequences outside the tRNA sequences. Despite the similarities of the attachment sites, the RP3 and VWB Int sequences did not display a high level of similarity.

For pSE101, it was speculated that the strand cleavage in $a t t B$ and attP occurs at one end of the identical segment, corresponding to the anticodon loop of the tRNA ${ }^{\mathrm{Th}}$ gene (Brown et al., 1994). For bacteriophage HP1 of Haemophilus influenzae it was reported that the HP1 integrase introduced a staggered cleavage with a 5-7 bp overlapping region. The overlap is again identical to the loop sequence of the tRNA ${ }^{\text {Leu }}$ anticodon arm (Hauser \& Scocca, 1992). Therefore, it is possible that the attB and attP crossover between the $S$. venezuelae chromosome and the VWB genome takes place at the $5^{\prime}$ end of the $45 \mathrm{bp}$ sequence, i.e. in the loop of the anticodon arm of the tRNA ${ }^{\text {Arg }}$ gene. The exact positions of cleavage by VWB Int, however, have not been located.

Two different inverted repeats were found overlapping the $5^{\prime}$ end of the identified common att sequence (Fig. 4). In att $B$ and att $L$, the inverted repeat is 5 bp long and consists of the stem of the tRNA ${ }^{\text {Arg }}$ anticodon arm. In attP and att $R$ a $7 \mathrm{bp}$ inverted repeat at about the same location is seen. The inverted repeats can function as recognition regions for the VWB integrase.

The meaning of the identical 5 bp box found in the att loci (Fig. 4) is not clear. In attB and attL, this box is localized in the stem of the tRNA D-arm but there is no obvious reason for the presence of this sequence in attP and $a t t R$. It could be a recognition site for the integrase or putative accessory proteins involved in the integration or excision process of the VWB genome.

\section{The use of attP and int to construct an integrative vector}

To prove the functionality and the requirement of the described int gene for site-specific recombination of attP with $a t t B$, the integrative vector pKT02 was constructed (Fig. 6a). This non-replicative plasmid carries the thiostrepton-resistance marker, the intact VWB int gene and the VWB attP locus. After transformation of $S$. venezuelae and S. lividans protoplasts with pKT02, thiostrepton-resistant clones could readily be obtained, suggesting that the pKT02 plasmid was incorporated into the chromosome. pKT02 transforms S. lividans 
TK24 more efficiently than it does $S$. venezuelae ETH14630 : per $\mu \mathrm{g}$ vector DNA 300-500 S. lividans TK24 and 10-50 S. venezuelae ETH14630 integrants could be obtained. The observed difference in transformation efficiency is probably more related to the difference in stability and regeneration capacity between $S$. lividans and $S$. venezuelae protoplasts and to a different restriction barrier in both strains, rather than to the efficiency of finding the att $B$ target sequence. In comparison with $\phi$ C31-based and pSAM2-based integrating vectors, the VWB-based vector pKT02 gave rather low amounts of integrants. Kuhstoss et al. (1991) reported that a $\phi$ C31-based and a pSAM2-based vector gave $3.8 \times 10^{6}$ and $6.4 \times 10^{3}$ Streptomyces ambofaciens BES2268 transformants per $\mu \mathrm{g}$ DNA, respectively. An RP3-derived integrating vector, however, transformed S. rimosus at a frequency of $10-100$ transformants per $\mu \mathrm{g}$ DNA (Gabriel et al., 1995).

Confirmation of the site-specific recombination of pKT02 in the S. venezuelae as well as in the S. lividans chromosome was obtained by Southern hybridization analysis (Fig. 6b). Total DNA of thiostrepton-resistant clones was isolated and digested with HindII. After electrophoresis and blotting, restriction fragments from the integrated DNA were identified with the digoxigenin-labelled $0.5 \mathrm{~kb}$ VWB BamHI-HindII fragment as probe. The results prove that the $2.3 \mathrm{~kb}$ VWB fragment used to construct the integrative pKT02 vector contains all information required for integration. pKT03, containing only the $0.7 \mathrm{~kb} B a m \mathrm{HI}-S p h \mathrm{I}$ VWB fragment, and pKT04, in which the $0.6 \mathrm{~kb}$ PstI fragment of pKT02 was deleted (Fig. 1), did not result in thiostreptonresistant colonies, proving that the int gene has to be functional to achieve site-specific recombination.

In S. lividans the recombination with the host chromosome seems to occur at a different region than the $\phi \mathrm{C} 31$ attachment locus. This is concluded from hybridization experiments of the VWB attP sequence with an $S$. coelicolor cosmid bank. The AseI-E fragment of the $S$. coelicolor genome that hybridizes with VWB attP $(\mathrm{H}$. Kieser, personal communication) does not contain the $\phi \mathrm{C} 31$ attachment sequence. As a consequence, the VWBbased integrative vector could be used in conjunction with $\phi \mathrm{C} 31$-based vectors, which might be useful for specific applications.

If the tRNA ${ }^{\mathrm{Arg}}$ gene, the recombination site for VWB integration in $S$. venezuelae, is conserved in other Streptomyces spp., it might be used as a general target site to introduce genes of interest. In this respect, we are currently using this vector for the transfer to $S$. lividans of genes encoding proteins of medical potential and we will further compare the stability of the recombinant strains and their yield to plasmid-based expression/ secretion systems.

\section{ACKNOWLEDGEMENTS}

This research was supported by VIS (Verkennende Internationale Samenwerking-VIS/95/10) and the Fund for Scientific Research (FWO). S.S. is a research fellow of iWT.
We thank Helen Kieser for carrying out the hybridization experiments with the $S$. coelicolor cosmid bank.

\section{REFERENCES}

Abremski, K. E. \& Hoess, R. H. (1992). Evidence for a second conserved arginine residue in the integrase family of recombination proteins. Protein Eng 5, 87-91.

Anné, J., Wohlleben, W., Burkardt, H. J., Springer, R. \& Püler, A. (1984). Morphological and molecular characterization of several actinophages isolated from soil which lyse Streptomyces cattleya or S. venezuelae. J Gen Microbiol 130, 2639-2649.

Anné, J., Van Mellaert, L. \& Eyssen, H. (1990a). Determination of the packaging capacity of bacteriophage VWB. J Bacteriol 172, 1129-1132.

Anné, J., Van Mellaert, L., Decock, B., Van Damme, J., Van Aerschot, A., Herdewijn, P. \& Eyssen, H. (1990b). Further biological and molecular characterization of actinophage VWB. J Gen Microbiol 136, 1365-1372.

Anné, J., Van Mellaert, L. \& Eyssen, H. (1990c). Optimum conditions for efficient transformation of Streptomyces venezuelae protoplasts. Appl Microbiol Biotechnol 32, 431-435.

Argos, P., Landy, A., Abremski, K., \& 9 other authors (1986). The integrase family of site-specific recombinases: regional similarities and global diversity. EMBO J 5, 433-440.

Bar-Nir, D., Cohen, A. \& Goedeke, M. E. (1992). tDNA ${ }^{\text {ser }}$ sequences are involved in the excision of Streptomyces griseus plasmid pSG1. Gene 122, 71-76.

Boccard, F., Pernodet, J.-L., Friedmann, A. \& Guérineau, M. (1988). Site-specific integration of plasmid pSAM2 in Streptomyces lividans and S. ambofaciens. Mol Gen Genet 212, 432-439.

Boccard, F., Smokvina, T., Pernodet, J.-L., Friedmann, A. \& Guérineau, M. (1989). The integrated conjugative plasmid pSAM2 of Streptomyces ambofaciens is related to temperate bacteriophages. EMBO J 8, 973-980.

Brasch, M. A., Pettis, G. S., Lee, S. C. \& Cohen, S. N. (1993). Localization and nucleotide sequences of genes mediating sitespecific recombination of the SLP1 element in Streptomyces lividans. J Bacteriol 175, 3067-3074.

Brown, D. P., Idler, K. B. \& Katz, L. (1990). Characterization of the genetic elements required for site-specific integration of plasmid pSE211 in Saccharopolyspora erythraea. J Bacteriol 172, 18771888.

Brown, D. P., Idler, K. B., Backer, D. M., Donadio, S. \& Katz, L. (1994). Characterization of the genes and attachment sites for site-specific integration of plasmid pSE101 in Saccharopolyspora erythraea and Streptomyces lividans. Mol Gen Genet 242, 185-193.

Chang, A. C. Y. \& Cohen, S. V. (1978). Construction and characterization of amplifiable multicopy DNA cloning vehicles derived from the p15A cryptic miniplasmid. J Bacteriol 134, 1141-1156.

Engler-Blum, G., Meier, M., Frank, J. \& Müller, G. A. (1993). Reduction of background problems in nonradioactive Northern and Southern blot analyses enables higher sensitivity than ${ }^{32} \mathrm{P}$ based hybridizations. Anal Biochem 210, 235-244.

Esposito, D. \& Scocca, J. J. (1997). The integrase family of tyrosine recombinases: evolution of a conserved active site domain. Nucleic Acids Res 25, 3605-3614.

Gabriel, K., Schmid, H., Schmidt, U. \& Rausch, H. (1995). The actinophage RP3 DNA integrates site-specifically into the putative tRNA ${ }^{\text {Arg }}(\mathrm{AGG})$ gene of Streptomyces rimosus. Nucleic Acids Res $23,58-63$. 
Hauser, M. A. \& Scocca, J. J. (1992). Site-specific integration of the Haemophilus influenzae bacteriophage HP1. Identification of the points of the recombinational exchange and the limits of the host attachment site. J Biol Chem 267, 6859-6864.

Hoeltke, H.-J., Schneider, S., Ettl, I., Binsack, R., Obermaier, I., Seller, M. \& Sagner, G. (1995). Rapid, highly sensitive detection of digoxigenin-labeled nucleic acids by improved chemiluminescent alkaline phosphatase substrates. Biochemica (Boehringer) 1, 17-20.

Hopwood, D. A., Bibb, M. J., Chater, K. F. \& 7 other authors (1985). Genetic Manipulation of Streptomyces: a Laboratory Manual. Norwich: John Innes Foundation.

Hopwood, D. A., Bibb, M. J., Chater, K. F. \& Kieser, T. (1987). Plasmid and phage vectors for gene cloning and analysis in Streptomyces. Methods Enzymol 153, 116-166.

Katz, E., Thompson, C. J. \& Hopwood, D. A. (1983). Cloning and expression of the tyrosinase gene from Streptomyces antibioticus in Streptomyces lividans. J Gen Microbiol 129, 2703-2714.

Kendall, K. \& Cullum, J. (1986). Identification of a DNA sequence associated with plasmid integration in Streptomyces coelicolor A3(2). Mol Gen Genet 202, 240-245.

Korn, F., Weingärtner, B. \& Kutzner, H. J. (1978). A study of twenty actinophages: morphology, serological relationship and host range. In Genetics of the Actinomycetales, pp. 251-270. Edited by E. Freerksen, I. Tarnok \& H. Thumin. Stuttgart \& New York: G. Fisher.

Kuhstoss, S. \& Rao, R. N. (1991). Analysis of the integration function of the Streptomycete bacteriophage $\phi$ C31. J Mol Biol 222, 897-908.

Kuhstoss, S., Richardson, M. A. \& Rao, R. N. (1991). Plasmid cloning vectors that integrate site-specifically in Streptomyces spp. Gene 97, 143-146.

Leschziner, A. E., Boocock, M. R. \& Grindley, N. D. F. (1995). The tyrosine- 6 hydroxyl of $\gamma \delta$ resolvase is not required for the DNA cleavage and rejoining reactions. Mol Microbiol 15, 865-870.

Marsh, J. L., Erfle, M. \& Wykes, E. J. (1984). The pIC plasmid and phage vectors with versatile cloning sites for recombinant selection by insertional inactivation. Gene 32, 481-485.

Matsuura, M., Noguchi, T., Yamaguchi, D., Aida, T., Asayama, M., Takahashi, H. \& Shirai, M. (1996). The sre gene (ORF469) encodes a site-specific recombinase responsible for integration of the R4 phage genome. J Bacteriol 178, 3374-3376.
Mazodier, P., Thompson, C. \& Boccard, F. (1990). The chromosomal integration site of the Streptomyces element pSAM2 overlaps a putative tRNA gene conserved among actinomycetes. Mol Gen Genet 222, 431-434.

Nunes-Duby, S. E., Kwon, H. J., Tirumalai, R. S., Ellenberger, T. \& Landy, A. (1998). Similarities and differences among 105 members of the Int family of site-specific recombinases. Nucleic Acids Res 26, 391-406.

Reiter, W.-D., Palm, P. \& Yeats, S. (1989). Transfer RNA genes frequently serve as integration sites for prokaryotic genetic elements. Nucleic Acids Res 17, 1907-1914.

Sambrook, J., Fritsch, E. F. \& Maniatis, T. (1989). Molecular Cloning: a Laboratory Manual, 2nd edn. Cold Spring Harbor, NY: Cold Spring Harbor Laboratory.

Sedlmeier, R., Werner, T., Kieser, H. M., Hopwood, D. A. \& Schmieger, H. (1994). tRNA genes of Streptomyces lividans: new sequences and comparison of structure and organization with those of other bacteria. J Bacteriol 176, 5550-5553.

Shirai, M., Nara, H., Sato, A., Aida, T. \& Takahashi, H. (1991). Sitespecific integration of the actinophage R4 genome into the chromosome of Streptomyces parvulus upon lysogenization. J Bacteriol 173, 4237-4239.

Sosio, M., Madon, J. \& Hütter, R. (1989). Excision of pIJ408 from the chromosome of Streptomyces glaucescens and its transfer into Streptomyces lividans. Mol Gen Genet 218, 169-176.

Sprinzl, M., Dank, N., Nock, J. \& Schön, A. (1991). Compilation of tRNA sequences and sequences of tRNA genes. Nucleic Acids Res $19,2127-2171$.

Vögtli, M. \& Cohen, N. (1992). The chromosomal integration site for Streptomyces plasmid SLP1 is a functional tRNA ${ }^{\mathrm{Tyr}}$ gene essential for cell viability. Mol Microbiol 6, 3041-3050.

Wertman, K. F., Wyman, A. R. \& Botstein, D. (1986). Host/vector interactions which affect the viability of recombinant phage lambda clones. Gene 49, 253-262.

Yang, W. \& Mizuuchi, K. (1997). Site-specific recombination in plane view. Structure 5, 1401-1406.

Yanisch-Perron, C., Vieira, J. \& Messing, J. (1985). Improved M13 phage cloning vectors and host strains: nucleotide sequences of the M13mp18 and pUC19 vectors. Gene 33, 103-119.

Received 1 April 1998; revised 11 August 1998; accepted 25 August 1998. 